\title{
Cooperative transport in a potassium ion channel
}

Jean-Fang Gwan, and Artur Baumgaertner

Citation: The Journal of Chemical Physics 127, 045103 (2007); doi: 10.1063/1.2756531

View online: https://doi.org/10.1063/1.2756531

View Table of Contents: http://aip.scitation.org/toc/jcp/127/4

Published by the American Institute of Physics

\section{Articles you may be interested in}

Free energy calculation from steered molecular dynamics simulations using Jarzynski's equality The Journal of Chemical Physics 119, 3559 (2003); 10.1063/1.1590311

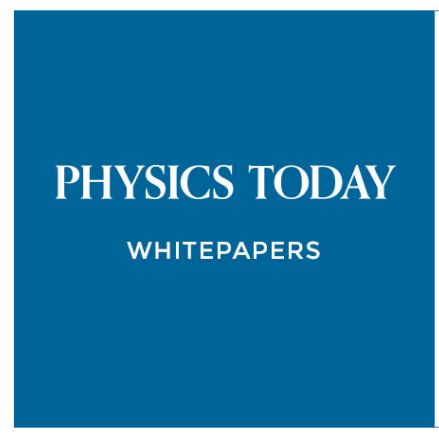




\title{
Cooperative transport in a potassium ion channel
}

\author{
Jean-Fang Gwan and Artur Baumgaertner \\ Institut für Festkörperforschung, Forschungszentrum Jülich, 52425 Jülich, Germany
}

(Received 23 March 2007; accepted 15 June 2007; published online 31 July 2007)

\begin{abstract}
Our current understanding of ion permeation through the selectivity filter of the KcsA potassium channel is based on the concept of a multi-ion transport mechanism. The details of this concerted movement, however, are not well understood. In the present paper we report on molecular dynamics simulations which provides new insights. It is shown that ion translocation is based on the collective hopping of ions and water molecules which is mediated by the flexible charged carbonyl groups lining the backbone of the pore. In particular, there is strong evidence for pairwise translocations where one ion and one water molecule form a bound state. We suggest a physical explanation of the observed phenomena employing a simple lattice model. It is argued that the water molecules can act as rectifiers during the hopping of ion-water pairs. (C) 2007 American Institute of Physics.
\end{abstract}

[DOI: $10.1063 / 1.2756531]$

\section{INTRODUCTION}

The presence of multiple potassium ions within the pore of $\mathrm{K}^{+}$channels seems to be a universal property among potassium channels. ${ }^{1}$ In particular, experimental results ${ }^{2,3}$ indicated that when the concentration of $\mathrm{K}^{+}$in solution is higher than about $20 \mathrm{mM}$, there are two ions in the pore, which serves as an "ion selectivity filter," at a given time, and that the two ions are expected to move in a concerted fashion between two "conductive states:" a $\mathrm{K}^{+}$-water-K $\mathrm{K}^{+}$-water occupancy of the four binding sites $S_{1}, S_{2}, S_{3}$, and $S_{4}$, in which ions bind at positions $\mathrm{S}_{1}$ and $\mathrm{S}_{3}$, and a water- $\mathrm{K}^{+}$-water- $\mathrm{K}^{+}$ configuration in which ions bind at positions $S_{2}$ and $S_{4}$. When the $\mathrm{K}^{+}$concentration in solution is much less than $20 \mathrm{mM}$, ion occupancy in the filter decreases, and the filter switches to a different conformation. ${ }^{4}$ These experimental findings form the basis of our current understanding of the high conduction rates in $\mathrm{K}^{+}$channels. The details of the molecular mechanism of the concerted movements of ions and water in the conductive state, however, are not well understood.

The ability of potassium channels to conduct $\mathrm{K}^{+}$ions at high levels near the limit of diffusion is usually described in terms of concerted mechanisms where several $\mathrm{K}^{+}$ions move simultaneously in a single file ${ }^{5}$ through the narrow pore. The translocations of the ions in the pore are thought to be initiated by a third ion which hops from the inner vestibule of the channel into site $\mathrm{S}_{4}$ at the entrance of the pore where it collides with the ion at $\mathrm{S}_{3}$ ("knock-on" mechanism"). This induces a concerted transition to a state in which the three ions occupy sites $S_{4}, S_{2}$, and $S_{0}$. This is then followed by departure of the outermost ions in $S_{0}$ to the extracellular side.

Employing molecular dynamics (MD) simulations on the basis of the X-ray structure of the KcsA channel, several computer simulations have provided useful insights into the structure-conductivity relationship. Ion mobilities and ion fluxes have been measured by Brownian dynamics simulations, ${ }^{7-10}$ and the potential profiles and free energy surface of ions inside the pore have been calculated employing molecular dynamics ${ }^{11-14}$ and Monte Carlo simulations. ${ }^{15}$

Comparing many classical transport theories in soft and solid state matter based on microscopic-mechanistic concepts with our present understanding of ion transport in potassium channels, the situation is not satisfactory, at least from a theoretical point of view. Several authors have proposed theories by employing the stochastic approach which intends to capture fluctuational characters of ionic transport. Theories have been developed where the ion transport through open channels takes place on the basis of the theory of reaction rates, ${ }^{16-18}$ where the pore is considered as a sequence of energy barriers over which the ion has to jump. The reaction-rate theory approach, however, was reported to be somewhat empirical in dealing with ionic permeation through channels. ${ }^{17}$ Other theories have been proposed by Lee and Sung ${ }^{19,20}$ where the effect of nonequilibrium fluctuations on ionic transport is included by the concept of localized ratchet, ${ }^{19}$ or where the coupling between ion transport and channel conformation under an applied transmembrane potential have been considered. ${ }^{20}$ Furthermore, the collective movements of the essential constituents of the pore of an ion channel, i.e., the backbone atoms, ions, and water molecules, have been described by employing the quasiparticle concept of "permions," 21 inspired by methods in solid state physics.

Unfortunately, most of the stochastic theories are unspecific in the sense that the underlying assumptions of the model cannot be directly related to the real situation. Therefore, the present study reports on an attempt to reconcile experimental facts with some microscopic concepts for the ion transport. Our approach is based on a common strategy in physics: the development of a theoretical model based on computer simulations. In our "computer experiment," we employed a technique, which is not new, ${ }^{22,23}$ and which is borrowed from the experimental technique of atomic force microscopy. By pulling out the outermost ion from the exit of the pore, we observed subsequent collective cooperative 


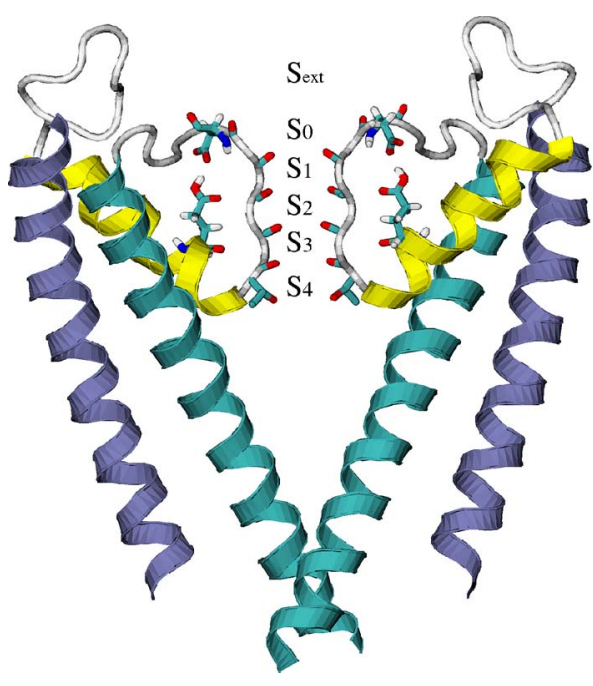

FIG. 1. The structure of the KcsA channel in ribbon representation, and the definition of the binding sites $\mathrm{S}_{j}$ in the selectivity filter.

movements of ions, water, and carbonyl groups lining the backbone of the pore. A detailed analysis of these movements leads to the development of a simple two-dimensional model of ion-water transport. The observations from our computer experiment seem to justify the permion concept $\mathrm{t}^{21}$ in the fact that the transport is performed by pairwise hopping of ion-water pairs mediated by the potential of the backbone atoms of the pore. This observation is also in accord with previous simulations of an artificial long channel. ${ }^{24}$ One important implication, which follows from the analysis of our computer experiment, is the fact that the water molecules located between the ions probably serve as rectifiers during the ion-water movements. This conjecture could explain the experimentally observed high transport rate in potassium ion channels.

\section{MATERIALS AND METHODS}

\section{A. Molecular model}

An atomic model of the KcsA channel (Fig. 1) embedded in the lipid bilayer was constructed for the molecular dynamics simulations. The atomic coordinates of the KcsA channel were taken from the crystallographic structures at $3.2 \AA$ (Ref. 25) [protein data bank (PDB) 1BL8] and $2.0 \AA$ (Ref. 4) (PDB 1K4C) resolutions. The channel protein is a tetramer composed of four identical monomers, M1, M2, M3, and M4, and each monomer contains 160 amino acid residues. Only the membrane part of the channel (residues 22-124) (Refs. 4 and 25) was resolved by experiments. The four monomers are denoted by M1, M2, M3, and M4. The $p \mathrm{H}$ environment of our simulations was set to 7. It has been suggested by both experimental and theoretical studies that Glu71, which is buried inside the protein, should be in a protonated (neutral) state. ${ }^{10,26-28}$ Other amino residues with ionizable side chains are located mostly at a solvent exposed area and were assigned to their ionized state. Other atomic charges of the selectivity filter have been proposed, ${ }^{29}$ which indicates the need of electronically polarizable force fields.

The selectivity filter is the most important structural

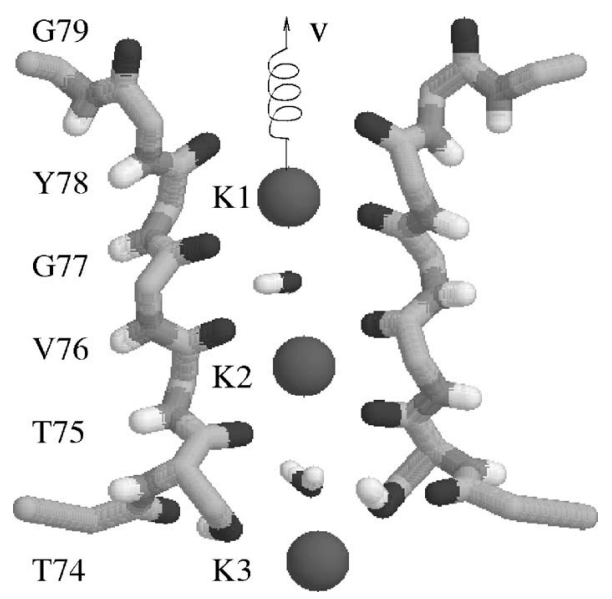

FIG. 2. Initial configuration of the selectivity filter including ions and water molecules. A symbolic spring is attached to the last ion.

component for the function of the KcsA channel. It is composed of the backbone atoms of the $\mathrm{K}^{+}$-channel signature sequence "TVGYG" with the carbonyl groups pointing towards the pore axis and the amide groups pointing away from the pore axis. The backbone carbonyl oxygens of the filter residues form five binding sites, denoted by $\mathrm{S}_{0}-\mathrm{S}_{4}$, as illustrated in Fig. 1. An extra site $S_{\text {ext }}$ located outside the extracellular mouth was identified in the KcsA crystallographic structure of higher resolution. ${ }^{4}$ When a $\mathrm{K}^{+}$ion is placed at a binding site in the filter, it is coordinated by eight carbonyl oxygens above and below it.

In the crystallographic data, ${ }^{4}$ which provide information of average ion configurations, three $\mathrm{K}^{+}$ions, which we denoted by $\mathrm{K} 1, \mathrm{~K} 2$, and $\mathrm{K} 3$, were found in the selectivity filter at sites $\mathrm{S}_{1}, \mathrm{~S}_{3}$, and $\mathrm{S}_{4}$, respectively. ${ }^{4}$ One water molecule, denoted by $\mathrm{W} 1$, was found at site $\mathrm{S}_{2}$ between $\mathrm{K} 1$ and $\mathrm{K} 2$. More recently, at concentrations of $\mathrm{K}^{+}$in solution higher than about $20 \mathrm{mM}$ it was found that the occupancy of the filter can have two "conductive" configurations: ${ }^{3}$ a $\mathrm{K}^{+}$-water$\mathrm{K}^{+}$-water configuration in which ions bind at positions $\mathrm{S}_{1}$ and $\mathrm{S}_{3}$, and a water- $\mathrm{K}^{+}$-water- $\mathrm{K}^{+}$configuration in which ions bind at positions $S_{2}$ and $S_{4}$. Since in the present work we are interested in the conductive properties of the selectivity filter, we have chosen the $\mathrm{K}^{+}$-water- $\mathrm{K}^{+}$-water configuration as the initial configuration of ouf KcsA model (Fig. 2). The pore of the KcsA channel forms a cavity of $10 \AA$ in diameter situated in the middle of the membrane. The empty space in the cavity and the $18 \AA$ long intravestibule of the channel were filled with TIP3P water molecules by a computer program and subsequently equilibrated with constrained protein coordinates. The pore of the channel contained 50 water molecules, approximately 40 of them were located in the cavity. The channel protein was then inserted into a phosphatidylcholine (POPC) lipid bilayer ${ }^{30,31}$ at a position where the $\mathrm{C}_{\alpha}$ of Asp80 was located around the average position of the phosphate groups of the upper lipid layer. Because of the asymmetric shape of KcsA, the upper and lower parts of the bilayer contained different numbers of lipids, 45 and 59, respectively. The membrane-protein system was then solvated by TIP3P model waters. Eleven $\mathrm{Cl}^{-}$were added to keep the system neutral. The final channel-membrane model con- 
tained 388 amino residues (97 residues in each monomer, 5908 atoms in total) and 104 lipid molecules, in total of 28928 atoms. Before production runs, the total system was equilibrated for $370 \mathrm{ps}$.

\section{B. Simulation methods}

Under physiological conditions the potassium channel provides a very efficient and ion selective valve, which allows the equilibration of the different potassium concentrations in the intracellular (high) and extracellular (low) space across the plasma membrane. Entering and exiting the selectivity filter of an ion channel is a probabilistic process depending on the different ion concentrations on each side of the membrane. According to the experimentally known throughput rate of the KcsA channel of about $10^{8}$ ions per second, the event of one exiting ion takes place on the average of every $10 \mathrm{~ns}$, which is a very rare event as compared to the typical time scale of MD simulations. Since we are not primarily interested in the event as such, but rather in the mechanism of ion movements, we imposed a weak perturbation on the system. The perturbation is achieved by displacing the last ion in the channel (Fig. 2) from its binding site $S_{1}$ to $\mathrm{S}_{\text {ext }}$. This method is the "steered" MD simulation method $^{22,23,32}$ used for studies on the dissociation processes of ligand-protein complexes. A harmonic force $F$ was applied to the ion $\mathrm{K} 1$ by attaching it to a symbolic spring. The pore axis of the channel was considered as a natural reaction coordinate of the ion translocation process. The spring was shifted towards the extracellular mouth with a constant velocity $v=0.015 \AA / \mathrm{ps}$ along the pore axis, as illustrated in Fig. 2. The mechanical (harmonic) force,

$$
F=k_{0}\left(z_{K 1}(t)-z_{0}(t)\right),
$$

was exerted on $\mathrm{K} 1$, where $k_{0}=140 \mathrm{pN} / \AA$ is the spring constant, $z_{\mathrm{K} 1}$ is the position of $\mathrm{K} 1$, and $z_{0}$ is the position of the end of the spring.

The simulation was carried out using the AMBER5.0 programs $^{33}$ on the supercomputer at the Forschungszentrum Jülich. The carbon atom together with its covalently bonded hydrogen atoms of the hydrocarbon chain of the lipid molecules were modeled as united atoms. ${ }^{31}$ The equation of motion was integrated using the Verlet leapfrog algorithm. Periodic boundary condition were applied to all three dimensions of the system. The simulation was done under the NPT condition. The temperature was kept at $300 \mathrm{~K}$ by applying the Berendsen coupling algorithm on the temperature scaling. ${ }^{34}$ The atoms of solute and solvent were coupled to separate heat bath to prevent the "cold solute/hot solvent" problem. ${ }^{35}$ The pressure was kept at 1.0 bar by using the anisotropic scaling with the coupling constant equal to 0.1 . The SHAKE constraint algorithm was employed to remove the stretching freedom of all bonds involving hydrogens. The particle mesh Ewald (PME) summation method ${ }^{36}$ with B-spline interpolation and fast Fourier transforms were used for the calculations of the electrostatic and Lennard-Jones interactions. The order of the B-spline interpolation was set to 4 , which is a cubic spline approximation, and the size of the charge grid was $\sim 1 \AA$ in each dimension. The direct sum tolerance of PME was 0.000 01. The $1-4$ van der Waals and the 1-4 electrostatic interactions (van der Waals/electrostatic interactions separated by only three covalent bonds) were both scaled ${ }^{37}$ by the factor 2.0. The equations of motion were integrated with a time step of $2 \mathrm{fs}$. The atomic coordinates were saved every $1 \mathrm{ps}$ and the velocity every $10 \mathrm{ps}$.

We have conducted three different MD simulations, denoted by SMD1, SMD2, and SMD3, each of them with different initial conformations of the whole system. The three different start configurations were taken from the trajectory of a $1 \mathrm{~ns}$ MD simulation of the system performed after the equilibration process as described above. In all three simulations the initial ion occupancy states of the selectivity filter were the same.

\section{RESULTS}

In order to support the reliability of our MD simulations, the next two subsections contain reports on the structural stability and fluctuations of the KcsA structure and, in particular, of its selectivity filter.

\section{A. Stability of channel structure}

The integral structural variation of the KcsA channel during the simulation can be described by the root-meansquare deviation (rmsd) of its structure. The rmsd of the structure was calculated by employing the "orthogonal transformation method." 38 By using this method, a rotation matrix, which optimizes the superposition between a given structure and a referenced one, is obtained, and the rmsd is equal to the minimum eigenvalue of this rotation matrix. The crystallographic structure in PDB file $1 \mathrm{~K} 4 \mathrm{C}$ is taken as the reference structure in the rmsd analysis. We have calculated the time average rmsd of $\mathrm{C}_{\alpha}$ atoms from the full structure and from the selectivity filter (from residue Glu71 to Asp80). The rmsd of $\mathrm{C}_{\alpha}$ atoms of the full structure and the filter region in two simulations, where $\mathrm{K} 1$ was pulled out of the filter, were almost of the same values, $1.73 \pm 0.09$ and $0.64 \pm 0.06 \AA$, respectively, as in a regular MD simulation, $1.71 \pm 0.13$ and $0.66 \pm 0.06 \AA$. This indicates that pulling K1 slowly out of the filter did not induce significant structural perturbations.

The stability of the structure of the selectivity filter is essential for the function of the KcsA channel. ${ }^{2,4}$ The structure of the selectivity filter is sustained by a delicate hydrogen-bonded network along the backbone of the filter. $^{25,39}$ From our simulation we found that the average bond pair distances remain close to the crystallographic data 4 with fluctuations less than $1.0 \AA$. In particular, the Glu71HE1-Asp80OD pair distance are very stable in all MD simulations, except in monomer M4 of SMD2. The bonding of the carboxyl carboxylate stabilizes other surrounded intrachain bonds. Note that the pair distances of Gly79HNAsp800D are long but the fluctuations are relatively small in the MD simulations.

In the crystallographic structure of high-salt concentration, a water molecule was observed buried in the filter area. ${ }^{4}$ It mediates the binding between the amide hydrogen of Gly79 and the carboxylate oxygen of Asp80. In our simula- 


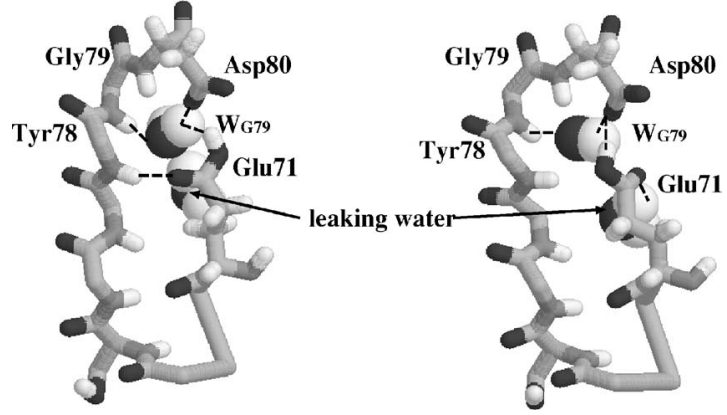

FIG. 3. The location of water molecule $\mathrm{W}_{\mathrm{G} 79}$. It mediates the bonding between Glu71-O-E1 and Tyr78-H-N. Two alternative conformations of the side chain of Glu71 are presented here. The switching motion of the side chain is induced by a water molecule leaking into the core part of the protein.

tions, a water molecule located at the predicted position is observed. The water molecule, denoted by $\mathrm{W}_{\mathrm{G} 79}$, had leaked into the channel protein during the simulation. In some of the channel structures of our simulations, more than one water molecules were observed leaking into the core part of the selectivity filter. The extra leaking waters induced the side chains of Glu71 to alter their orientations, which perturbed the selectivity filter structure, as shown in Fig. 3.

Although our simulation system is actually in the "lowsalt" condition, the filter structure in most of the simulations shows good agreement with the high-salt structure, except during the event of the malfunction of the filter as observed in simulation SMD2 (see below).

\section{B. Dynamics of $\mathrm{K}^{+}$and water in the selectivity filter}

Among the three MD simulations, the dynamics of ions and water molecules in simulation SMD1 and SMD3 have similar features, which are based on the preserved structural integrity of the selectivity filter, in particular, with regard to the correct orientation of the carbonyl groups. We present here the data of SMD1 only. The upper part of Fig. 4 shows the $z$ trajectories of the potassium ions K1, K2, and K3 (red) and the water molecules W1, W2 (cyan), W3 (blue), and W4 (green) inside the filter. The figure shows also the trajectories of the carbonyl oxygens of the selectivity filter residues T-TV-G-Y-G (black lines) as marked at the right margin. The results show that at time $139 \pm 3$ ps a translocation of $\mathrm{K} 1$ from binding site $S_{1}$ to site $S_{0}$ started to take place, which was induced by the harmonic pulling force acting on $\mathrm{K} 1$. This transition was completed within less then 6 ps. Almost simultaneously, the neighboring water molecule $\mathrm{W} 1$ followed the preceding $\mathrm{K} 1$ from $\mathrm{S}_{2}$ to $\mathrm{S}_{1}$. At $\approx 145 \pm 3$ ps the next ion $\mathrm{K} 2$ followed from $\mathrm{S}_{3}$ to $\mathrm{S}_{2}$. On a time scale of about $6 \mathrm{ps}$, the whole complex of K1-W1-K2 had performed a "concerted movement" by one binding site. The ion K2 and the water W1 remained at their new sites until the end of the simulation in both SMD1 and SMD3. K1 exited the channel to the external binding site $S_{\text {ext }}$ at about 350 ps. During the translocations of the water molecules, their orientations with respect to the symmetry axis of the filter changed significantly and seemed to be correlated not only with the neighboring ions, but also with the carbonyl groups lining the
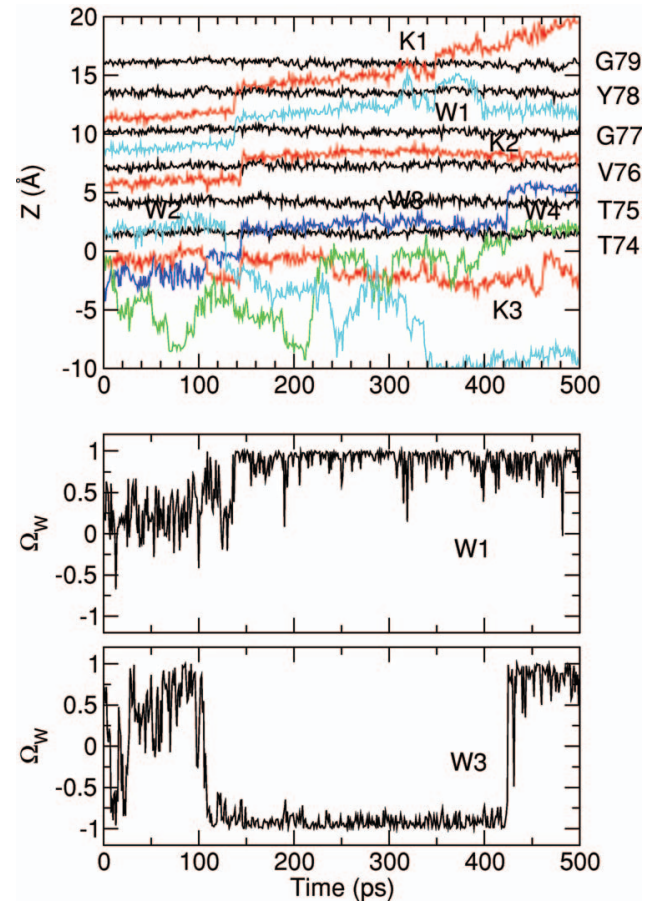

FIG. 4. (Color) Upper part. Ion, water, and carbonyl trajectories in SMD1. The $z$ trajectories of ions $\mathrm{K} 1, \mathrm{~K} 2$, and $\mathrm{K} 3$ (red); water molecules W1, W2 (cyan), W3 (blue), and W4 (green); and the carbonyl oxygens of the selectivity filter residues T-T-V-G-Y-G (black) are shown. Lower part: Orientational angle $\Omega_{\mathrm{W}}$ with respect to the pore axis for waters W1 and W3. The times of the translocation events are $137 \pm 3$ and $145 \pm 3$ ps for $\mathrm{W} 1$ and K2, respectively.

selectivity filter. The orientation of a water molecule with respect to the $z$ axis is described by the angle $\Theta_{\mathrm{W}}$ of its water dipole according to $\Omega_{\mathrm{W}} \equiv \cos \Theta_{\mathrm{W}}=z_{\mathrm{O}}-z_{\mathrm{HH}} / r_{\mathrm{O}-\mathrm{HH}}$, where $z_{\mathrm{O}}$ is the $z$ coordinate of the water oxygen, $z_{\mathrm{HH}}$ is the $z$ coordinate of the center of the two hydrogen atoms, and $r_{\mathrm{O}-\mathrm{HH}}$ is the distance between water oxygen and the center of the two hydrogens. The time variations of $\Omega_{\mathrm{W}}$ for $\mathrm{W} 1$ and $\mathrm{W} 3$ (Fig. 4) indicate a strong correlation between ion-water translocation and water orientation. While moving from $S_{2}$ to $S_{1}$, W1 became oriented by $\mathrm{K} 1$ and switched from 0 to +1 at the first translocation at $140 \mathrm{ps}$. Since W3 was bound to the K3 when it entered the selectivity filter from the cavity at $110 \mathrm{ps}$, its orientation switched from a fluctuating value to $\Omega_{\mathrm{W}}=-1$ which remained stable until 430 ps. Then it became reoriented oppositely due to the $\mathrm{K} 2$ during its translocation to $\mathrm{S}_{3}$

A similar pattern of transition as compared to SMD1 was also observed in the simulation SMD3. A concerted movement of K1-W1-K2 occurred at about 160 ps and the time delay between the translocations of $\mathrm{K} 1$ and $\mathrm{W} 1-\mathrm{K} 2$ was less than 1 ps. In simulations SMD1 and SMD3, the site $S_{3}$ became occupied by a water molecule after 250 and 200 ps, respectively. Similar coupled translocation has been observed in other simulations. ${ }^{39,40}$

\section{Coupling of carbonyl groups with water and $\mathbf{K}^{+}$}

Previous MD simulations studies ${ }^{39,40}$ have shown that during the motions of the ions subtle changes in the backbone conformation of the selectivity filter can appear, which indicates towards a coupling between ion permeation and 


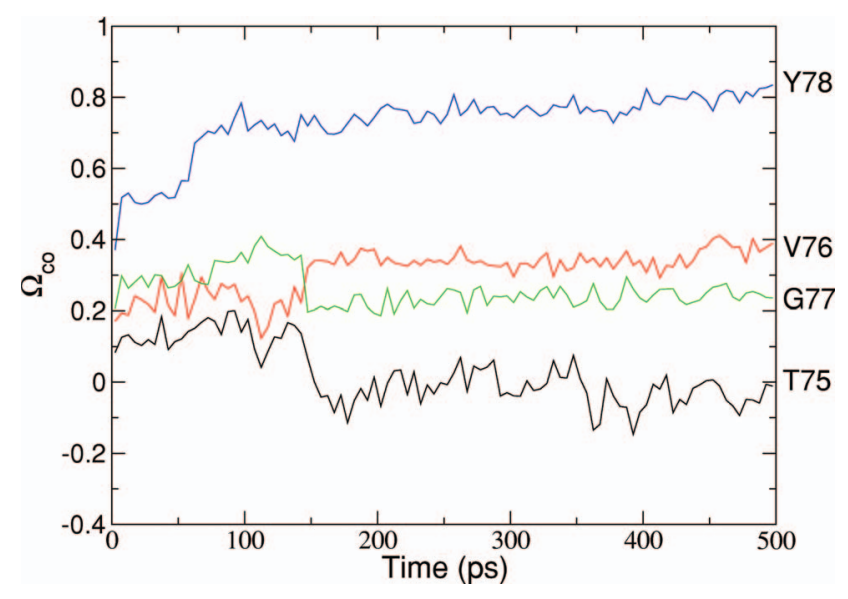

FIG. 5. (Color) Time variation of the orientation, $\Omega_{\mathrm{CO}}$, of the carbonyl groups of TVGY.

positional changes of the carbonyl groups. Similar findings can be reported from our simulations. It is observed that the concerted movement of $\mathrm{K}^{+}$and water is accompanied by a correlated motion of the carbonyl groups lining the selectivity filter, which is analyzed in the following.

The trajectories of the carbonyl oxygens of the sequence T-V-G-Y, which are shown in Fig. 4, exhibit a slight displacement downwards the channel axis during the concerted movements of the potassium ions and the water molecules. This is basically due to a reorientation of the $\mathrm{C}-\mathrm{O}$ dipole of the carbonyls. The orientation can be defined by

$$
\Omega_{\mathrm{CO}} \equiv \sin \Theta_{\mathrm{CO}}=z_{\mathrm{O}}-z_{\mathrm{C}} / r_{\mathrm{O}-\mathrm{C}},
$$

where $z_{\mathrm{O}}$ is the $z$-coordinate of the carbonyl oxygen, $z_{\mathrm{C}}$ is the $z$-coordinate of the carbonyl carbon, and $r_{\mathrm{O}-\mathrm{C}}$ is the distance between them. The simulation data of $\Omega_{\mathrm{CO}}$, averaged in parcels of 5 ps, are shown Fig. 5 for each of the carbonyls of the sequence T-V-G-Y. In variance with the observed translocations in Fig. 4, the orientations of the carbonyls changed at these transitions as well (Fig. 5). This observation indicates that the translocation of ions requires not only a subtle cooperation between $\mathrm{K}^{+}$and water, but also between the backbone atoms of the selectivity filter and $\mathrm{K}^{+}$and water. This will be discussed in more detail in Sec. IV.

\section{Malfunction of the selectivity filter}

The simulations SMD1 and SMD3 are examples of successful ion-water translocations through the selectivity filter. However, from the point of view of statistical mechanics, it must be expected that with a certain probability, a translocation event cannot be completed successfully. One of these events had been observed during the simulation SMD2. Since it is very instructive to understand which molecular events may prohibit or at least retard translocation, the molecular features of an uncomplete translocation are presented in the following subsection. The $z$ trajectories of the three $\mathrm{K}^{+}$ ions and water molecules $\mathrm{W} 1-\mathrm{W} 5$ in simulation SMD2 are shown in Fig. 6.

The first translocation of $\mathrm{K} 1$ from site $\mathrm{S}_{1}$ to $\mathrm{S}_{0}$ occurred at $200 \mathrm{ps}$. Although starting from the same initial conductive state configuration K1-W1-K2-W2 as in SMD1 and SMD3,
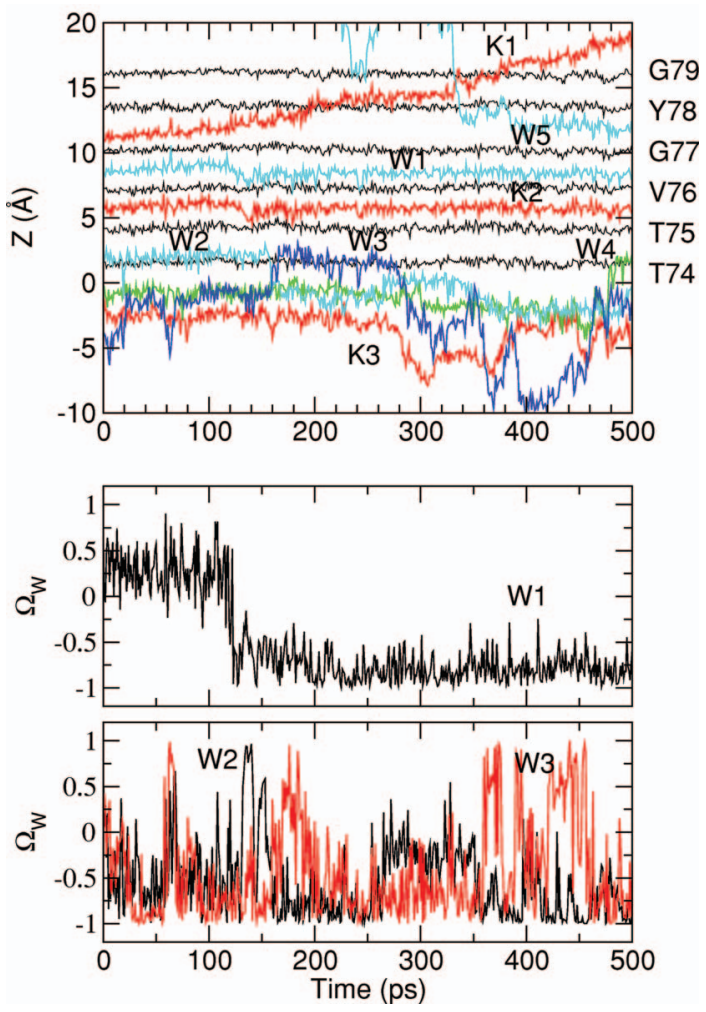

FIG. 6. (Color) The $z$ trajectories of ions $\mathrm{K} 1, \mathrm{~K} 2$, and $\mathrm{K} 3$ (red); water molecules W1, W2 (cyan), W3 (blue), W4 (green), and W5 (cyan); and the carbonyl oxygens of the selectivity filter residues T-T-V-G-Y-G (black) are shown. Lower part: Orientational angle $\Omega_{\mathrm{W}}$ with respect to the pore axis for water $\mathrm{W} 1$ and $\mathrm{W} 3$.

the trajectories of SMD2 differ such that no concerted movement was observed in this simulation, and $\mathrm{W} 1$ and $\mathrm{K} 2$ remained in their initial locations at sites $\mathrm{S}_{2}$ and $\mathrm{S}_{3}$. The reason for the absence of successful translocation was the deformation of the structure of the selectivity filter during the simulation. As shown in Fig. 7, the Val76-Gly77 backbone of monomer M4 twisted such that the $\mathrm{C}=\mathrm{O}$ of Val76 of $\mathrm{M} 4$ pointed away from the pore axis and the $\mathrm{N}-\mathrm{H}$ group of Gly77 turned inwards pointing towards the pore axis. Similar observations have been reported recently from other MD simulations of KcsA in its conductive state. ${ }^{39}$ The twist of the

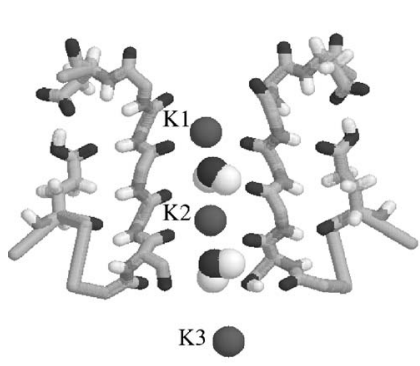

(a)

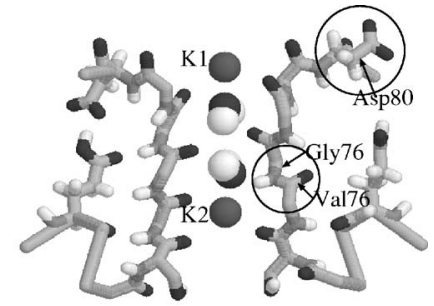

${ }^{\mathrm{K} 3} \mathrm{C}$

(b)
FIG. 7. The alternative conformations of the selectivity filter in SMD2. (a) The initial conformation of the selectivity filter. Only monomers M2 and M4 are shown here. (b) The alteration of the selectivity filter conformation. The amide hydrogen of Gly77 in M4 turns towards the pore axis and bound with a water molecule. The side chain of Asp80 changes its orientation and the binding between Glu71 and Asp80 is broken up. 


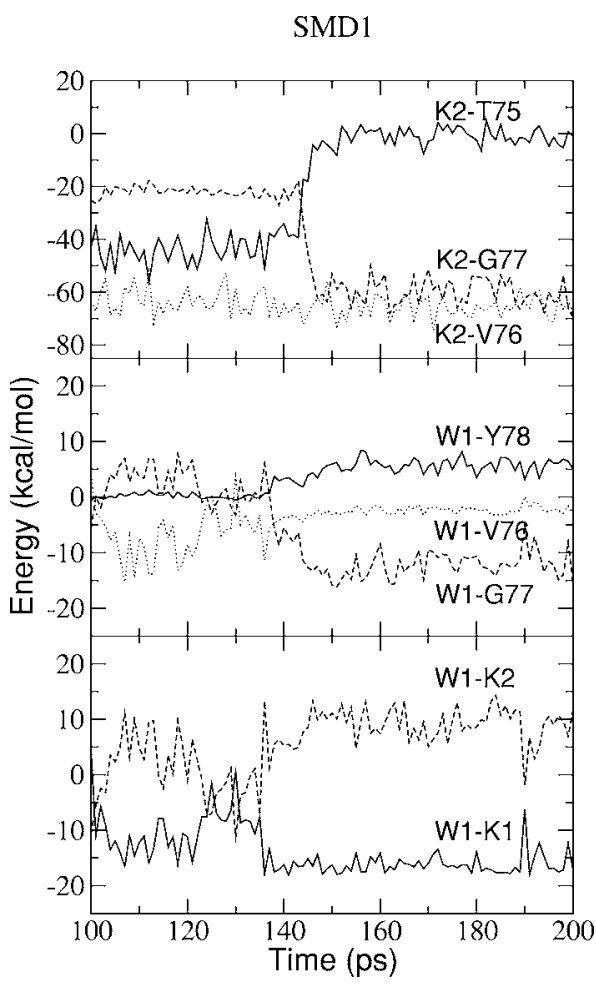

FIG. 8. The variation of the interaction energy before and after the first translocation in SMD1. The interaction energy between K2 and the carbonyl groups of Thr75, Val76, and Gly77 (top), between W1 and the carbonyl groups of Val76, Gly77, and Tyr78 (middle), and between W1 and K1 and K2 (bottom), are shown. The energy calculation includes the whole residue.

Val76-Gly77 backbone was induced by the tumbling motion of W1. Because of the displacement of K1, W1 had to adjust itself to a different conformation to minimize the energy. As shown in Fig. 6, the $\Omega_{\mathrm{W}}$ of $\mathrm{W} 1$ fluctuated between 0 and +1 during the time interval of $125-130 \mathrm{ps}$. This leads the Val76Gly77 backbone of M2 to twist for a short time and then turned back to the original orientation. Later it caused the same segment of M4 to twist. The oxygen atom of W1 and the amide hydrogen of Gly77 of M4 formed a stable hydrogen bond and they remained like this for the rest of the simulation. The binding of $\mathrm{W} 1$ and the amide hydrogen of Gly 77 blocked the selectivity filter and thus W1 and K2 remained at the same binding sites until the end of the simulation. The observed distortion of the selectivity filter is very similar to experimentally observed deformations of the KcsA channel at low-salt concentration. ${ }^{4}$ The structure of the distorted filter may return to its correct one, if a $\mathrm{K}^{+}$would enter the selectivity filter from the extracellular mouth. This ion may activate $\mathrm{W} 1$ again in such a way that $\mathrm{W} 1$ unbinds from the amide hydrogen of Gly77. Since at low-salt concentration, the occurrence of such an event is less probable, the system must be expected to remain in a malfunctional conformation for a long time.

\section{E. Energetics of the translocation process}

The interaction energies between the $\mathrm{K}^{+}$ions, the water molecules, and the residues, which are involved in the concerted movement, are shown in Fig. 8. The interaction energy is the sum of the contributions from electrostatic and van der Waals potentials. As expected, during the translocation of ions and water within the time interval $140-150 \mathrm{ps,}$ as shown in Fig. 4, the concomitant changes of interaction energies are significant and rather sharp. Most importantly, the total change is at almost no cost of energy. During the translocation of $\mathrm{K} 2$ from $\mathrm{S}_{3}$ (between Thr75 and Val76) to $\mathrm{S}_{2}$ (between Val76 and Gly77) the changes are both approximately $40 \mathrm{kcal} \mathrm{mol}^{-1}$ (Fig. 8): from -40 to $0 \mathrm{kcal} \mathrm{mol}^{-1}$ for the K2-Thr75 interaction, and from -20 to $-60 \mathrm{kcal} \mathrm{mol}^{-1}$ for the K2-G77 interaction. The interaction between K2 and Val76 remains approximately constant.

A similar scenario of transitions is observed for the interaction between W1 and the filter residues. Since the dipole orientation of $\mathrm{W} 1$ is most of the time such that its oxygen points upward, $\Omega_{\mathrm{W}} \geqslant 0$ (Fig. 4), the interactions between W1 and the residues and the $\mathrm{K}^{+}$below are attractive, while between $\mathrm{W} 1$ and the residues and the $\mathrm{K}^{+}$above are repulsive. After the translocation, when $\mathrm{W} 1$ had moved to $S_{1}$ and were surrounded by the carbonyl groups of Gly77 and Tyr78, its water dipole oriented entirely upward $\left(\Omega_{\mathrm{W}}=1\right)$ and bound to $\mathrm{K} 1$. The interaction energy between $\mathrm{W} 1$ and $\mathrm{K} 1$ became $-18 \mathrm{kcal} \mathrm{mol}^{-1}$, and between $\mathrm{W} 1$ and $\mathrm{K} 2,+10 \mathrm{kcal} \mathrm{mol}^{-1}$. The interaction energy between a water and residues away from it is zero (e.g., between W1 and Val76 in Fig. 8).

From the analysis of the interaction energies, we conclude that rather than the long-range electrostatic ion-ion repulsion, the concerted movement is more dominated by the shorter-range dipolar interaction involving the two types of polar groups, namely, the water molecule and the carbonyl group. When a potassium ion is far from a carbonyl group, the electrostatic interaction between them is slightly repulsive $^{33}$ almost independent of the carbonyls' orientation, whereas at nearest-neighbor positions the interaction can be repulsive or attractive depending on the orientations of the carbonyl groups. This indicates the importance of nearestneighbor coupling among ions, water, and carbonyls.

\section{DISCUSSIONS}

The ability of potassium channels to conduct several $\mathrm{K}^{+}$ ions simultaneously through the narrow pore is usually described in terms of concerted mechanisms. ${ }^{5,6}$ In the following we discuss the dynamics of the pore potential and how this correlates with the interaction between ions and water molecules inside the pore. Collective motions of ions, water molecules, and carbonyls lining the pore are explained employing a simple two-dimensional model.

\section{A. Dynamics of the pore potential}

We define two types of pore potentials, $U_{\mathrm{K}}(z)$ and $U_{\mathrm{W}}(z)$, which are the interactions between the whole channel protein and a single $\mathrm{K}^{+}$ion and a single water molecule, respectively. The pore potentials along the pore axis at different times $t$ are shown in Fig. 9. The pore potentials were calculated for times $t$ which are close to the translocation events of $\mathrm{K} 1, \mathrm{~W} 1$, and $\mathrm{K} 2$, as reported in the previous section (Fig. 4). The onset of the translocations of K1 and W1 was approximately at $139 \pm 3 \mathrm{ps}$, the translocation of K2 started around $145 \pm 3$ ps. The pore potential at each position 

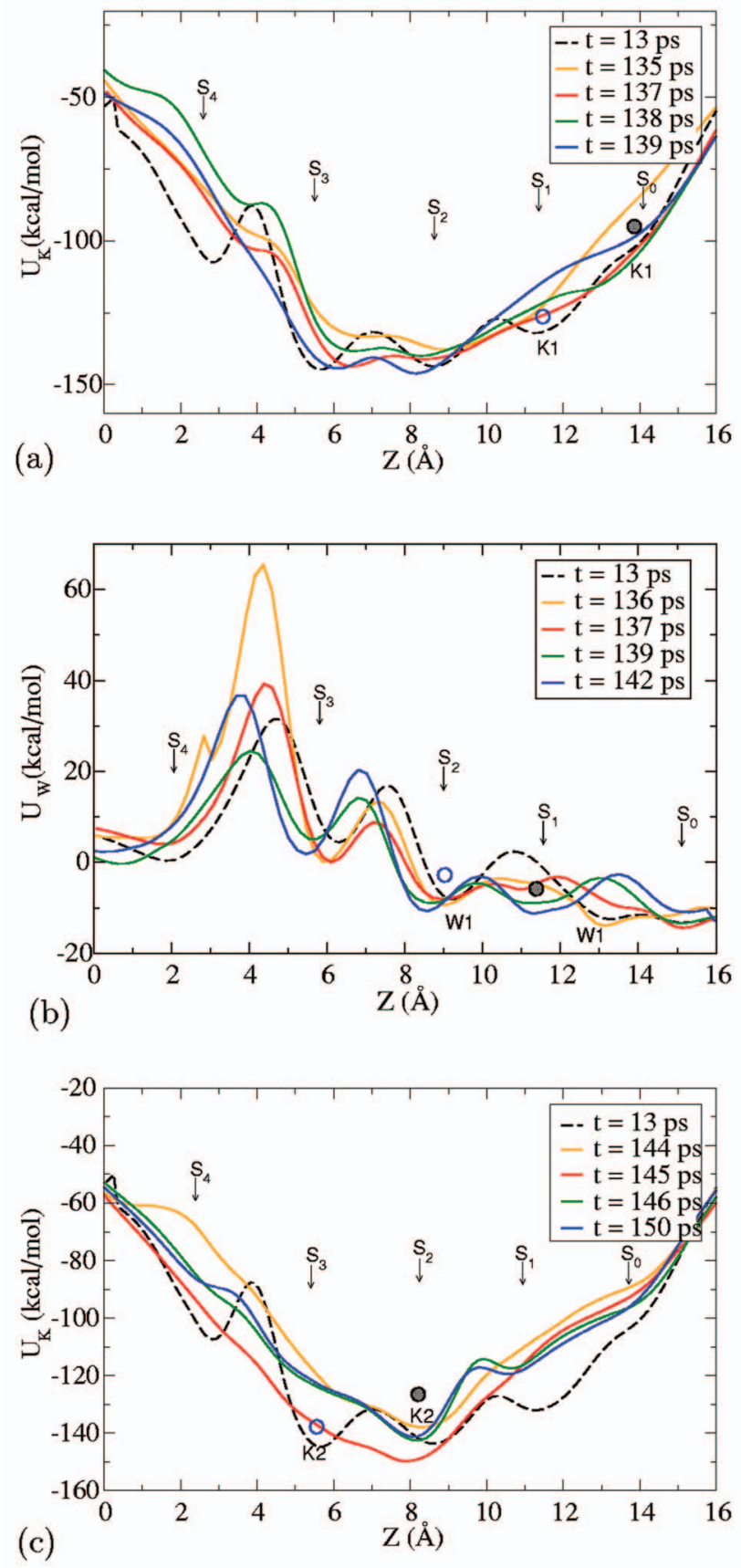

FIG. 9. (Color) The pore potentials $U(z)$ at different times $t$ : [(a) and (c)] $U_{\mathrm{K}}(z)$ for one $\mathrm{K}^{+}$ion and (b) $U_{\mathrm{W}}(z)$ for one water molecule. The open and full circles illustrate the average positions of the particles before and after their translocation, respectively.

$z$ was determined by calculating the electrostatic and van der Waals interactions between the particle (water or ion) and the ion channel. The energy minimum in the $x-y$ plane of the pore at fixed $z$ was estimated by Monte Carlo methods. Figure 9(a) shows the development of the pore potential $U_{\mathrm{K}}$ of a potassium ion before the translocation of $\mathrm{K} 2$ at $t=145 \mathrm{ps}$. The figure shows that the temporal change of the pore potential is correlated with the translocation of the exiting $\mathrm{K} 1$ from $\mathrm{S}_{1}$ to $\mathrm{S}_{0}$ at $t=139 \mathrm{ps}$. Initially $(t=13 \mathrm{ps}), \mathrm{K} 1$ is located at the potential minimum at site $S_{1}$. Due to the applied force on $\mathrm{K} 1$ in the positive $z$ direction, the ion exhibits biased positional fluctuations towards the exit (Fig. 4). This influ- ences the orientation of the neighboring carbonyl groups, in particular, Y78, which changes its orientation such that its oxygen atom follows the movement of $\mathrm{K} 1$, and hence the orientation $\Omega_{\mathrm{CO}}$ becomes positive, as shown in Fig. 5. This leads to a deformation of the pore potential, as shown in Fig. 9(a). At $t=139 \mathrm{ps}, \mathrm{K} 1$ translocates to its new site at $\mathrm{S}_{0}$; where $\mathrm{K} 1$ resides there until $t=350 \mathrm{ps}$ when it exits the pore.

Figure 9(b) shows the development of the pore potential for a single water molecule, $U_{\mathrm{W}}$, in the time interval 136 ps $\leqslant t \leqslant 142$ ps while K1 performs its translocation from $\mathrm{S}_{1}$ to $\mathrm{S}_{0}$. Since the water molecule $\mathrm{W} 1$ follows the preceding $\mathrm{K} 1$ from $\mathrm{S}_{2}$ to $\mathrm{S}_{1}$, it is of interest to examine whether the concomitant K1-induced change of the water's pore potential favors the translocation of W1. Indeed, before its transition at $139 \mathrm{ps}, \mathrm{W} 1$ is located at the minimum of $\mathrm{S}_{2}$. Presumably due to the translocation of $\mathrm{K} 1$, the pretransitional minimum of $U_{\mathrm{W}}$ at $\mathrm{S}_{2}$ gradually becomes shallower at the expense of the minimum at $S_{1}$ which becomes deeper with increasing time. At about $t=139 \mathrm{ps}$, the potential barrier between $\mathrm{S}_{2}$ and $\mathrm{S}_{1}$ is only a few $\mathrm{kcal} / \mathrm{mol}$, which is small compared to the $\mathrm{K} 1-\mathrm{W} 1$ interaction energy of the order of $-15 \mathrm{kcal} / \mathrm{mol}$ (Fig. $8)$. Hence the K1-induced change of the pore potential together with the high binding energy of K1-W1 causes the pairwise translocation of K1-W1 at $t=139 \mathrm{ps}$.

Figure 9(c) shows the development of $U_{\mathrm{K}}$ after the translocation of K1 and W1. Within the time interval of $144 \mathrm{ps}$ $\leqslant t \leqslant 150$ ps the translocation of $\mathrm{K} 2$ takes place. Before its translocation ( $t=13 \mathrm{ps}), \mathrm{K} 2$ is located in its potential minimum at $\mathrm{S}_{3}$. With increasing time the potential barrier between $S_{3}$ and $S_{2}$ gradually disappears. At about $t=145 \mathrm{ps}$, $\mathrm{K} 2$ moves to the binding site $\mathrm{S}_{2}$. The new position of K2 at $\mathrm{S}_{2}$ is stabilized by the subsequent decrease of the pore potential at this site relative to the potentials at the neighboring binding sites. The bistability of the local double-well potential at sites $S_{3}$ and $S_{2}$ is presumably caused by the preceding translocation event of the pair K1-W1. This kind of bistable switching from $S_{3}$ to $S_{2}$ is at least locally at no cost of energy, which is also consistent with the data in Fig. 8, where only the nearest-neighbor interactions during the translocation are considered.

\section{B. A two-dimensional model}

In order to develop a physical explanation for the observed concerted movements of ions and water it is useful to consider the following simple two-dimensional model. The pore potentials $U_{\mathrm{K}}(z)$ and $U_{\mathrm{W}}(z)$ are described approximately by the corresponding two-dimensional periodic "lattice potentials" $\widetilde{U}_{\mathrm{K}}$ and $\widetilde{U}_{\mathrm{W}}$. The geometry of such a "lattice" model, which approximates the geometry of the selectivity filter, is depicted in Fig. 10. The model consists of two parallel rods at a distance of $7.1 \AA$. One rod represents one peptide backbone of the selectivity filter. At each rod six thin sticks of length $1.24 \AA$, representing the carbonyl groups of the sequence T-V-G-Y-G, are attached at a distance of $3 \AA$ from each other. This is the distance between the carbonyl groups lining the selectivity filter and defines the periodicity of the pore potential. Each stick carries a charge of -0.5 e.u. at its tip and +0.62 e.u. at its junction with the backbone. 


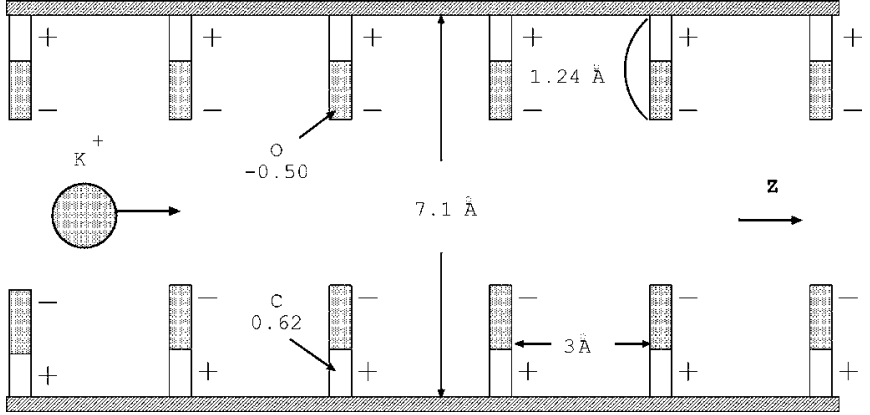

FIG. 10. Illustration of the geometry of a simple two-dimensional model of the KcsA selectivity filter.

Each carbonyl group is flexible at its junction and may exhibit a tilt angle $\theta$ with respect to the normal of the backbone. The lattice potentials for a single ion and for a single water molecule at certain tilt angles $\theta$ of a single cabonyl group are shown in Figs. 11(a) and 11(b), respectively. The potential contains electrostatic and van der Waals interactions. The numerical calculations show that the lattice potentials for an ion and a water molecule differ significantly. At $\theta=0$, the energy barrier for the ion is $\Delta \widetilde{U}_{\mathrm{K}} \approx 15 \mathrm{kcal} / \mathrm{mol}$ $\left(24.38 k_{B} T\right)$, whereas for the water molecule $(\Omega=1) \Delta \tilde{U}_{\mathrm{W}}$ $\approx 24 \mathrm{kcal} / \mathrm{mol}\left(39 k_{B} T\right)$. At equilibrium the barrier heights are sufficient to keep both ions and water trapped at their binding sites.

One important fact for the understanding of the efficiency of the collective movements in the pore, in particular, the translocation of ion-water pairs (Fig. 4), is the commensurability of the periodicity of the pore potential, $\approx 3 \AA$, with the distance of potential minimum of ion-water interaction, $\approx 2.7 \AA$. The classical potential energy $U_{\mathrm{KW}}(\Delta z)$ between a single ion and the three atoms of a single TIP3P water molecule, including Coulomb and van der Waals interactions, as function of the relative position between the centers of ion

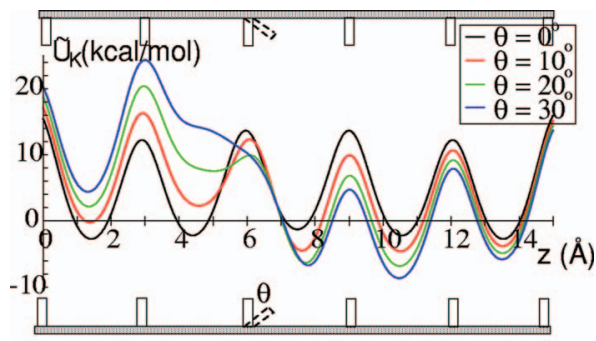

(a)

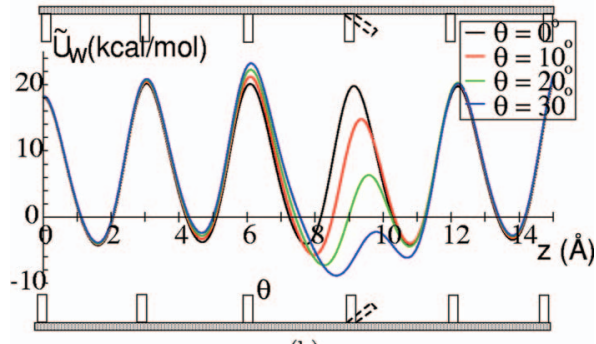

(b)

FIG. 11. (Color) The lattice potentials: (a) $\tilde{U}_{\mathrm{K}}(z)$ for a single ion and (b) $\tilde{U}_{\mathrm{W}}(z)$ for a single water molecule.

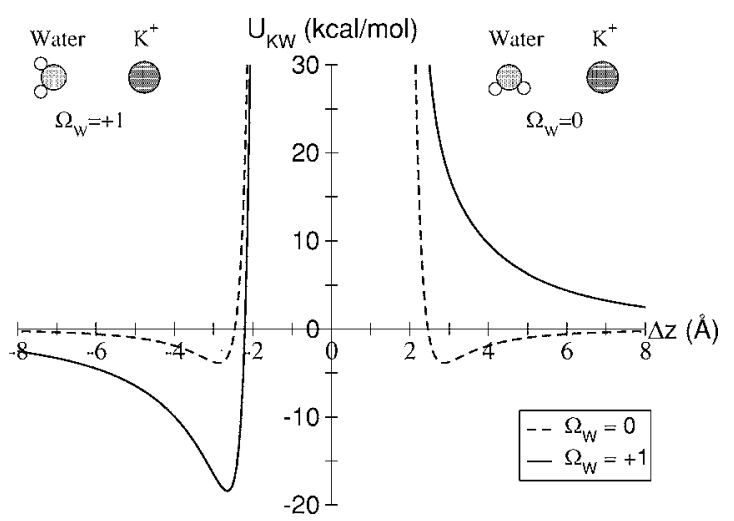

FIG. 12. The interaction energy between a $\mathrm{K}^{+}$ion and a water molecule with different orientations $\Omega_{\mathrm{W}}$ as a function of their relative position $\Delta z$ $=z_{\text {water }}-z_{\text {ion }}$. The "polarized bound state" at $2.65 \AA$ has an energy of $-18.08 \mathrm{kcal} / \mathrm{mol}\left(30 k_{B} T\right)$.

and oxygen, $\Delta z=z_{\text {water }}-z_{\text {ion }}$, is shown in Fig. 12. Electronic polarizabilities of ion and water molecules are not included. For $\Omega_{\mathrm{W}}=0$, the potential is symmetric with respect to $\Delta z$ $>0$ and $\Delta z<0$ (Fig. 12, broken line), whereas for $\Omega_{\mathrm{W}}=1$ (Fig. 12, full line) the interaction is attractive for $\Delta z<0$ and repulsive for $\Delta z>0$. The absolute minimum ("polarized bound state") of $U_{\mathrm{KW}}=-18.4 \mathrm{kcal} / \mathrm{mol}\left(30 k_{B} T\right)$ is for $\Omega_{\mathrm{W}}$ $=1$ at $\Delta z=-2.65 \AA$. The attractive part of the ion-water dipole interaction is given approximately ${ }^{41}$ by $U_{\mathrm{KW}}(z)$ $=\left(-69 \mu \Omega_{\mathrm{W}}\right) / \Delta z^{2} D(\mathrm{kcal} / \mathrm{mol})$, where $\mu$ is the dipole moment of water in debyes, $z$ is the distance in angstroms from the dipole center to the ion, and $D$ is the dielectric constant. For example, for $\Omega_{\mathrm{W}}=1, D=1, \mu=2.34$, and $\Delta z=2.65$, the energy is $U_{\mathrm{KW}}=18.08$. These simple calculations show that at equilibrium the potassium ions and the water molecules are located at the minima of the pore potential ("binding sites") forming pairwise "bound states" due to ion-water attraction.

Consider now the situation where the ion $\mathrm{K} 1$ at $\mathrm{S}_{1}$ moves to site $S_{0}$, towards right (Fig. 11). The moving ion induces a tilt $\theta$ at its left and right carbonyl groups due to the Coulomb attraction between $\mathrm{K}^{+}$and $\mathrm{CO}^{-}$, which was observed in our MD simulations (Fig. 5). The potential $\widetilde{U}_{\mathrm{K}}(z)$ changes, becomes locally bistable, and facilitates the movement of the ion.

One important consequence of the ion-induced local tilting of the carbonyls is the fact that the pore potential $\widetilde{U}_{\mathrm{W}}(z)$ of the neighboring water molecule W1 at site $S_{2}$ is changed as well. This is illustrated in Fig. 11(b) for $\Omega_{\mathrm{W}}=1$. Due to the ion-induced tilt of the carbonyls, the potential for the water molecule becomes bistable and the energy barrier towards $S_{1}$ is reduced from $\Delta \tilde{U}_{\mathrm{W}} \approx 39 k_{B} T$ to $10 k_{B} T$ at $\theta=30^{\circ}$. Since the ion-water attraction is about $U_{\mathrm{KW}}=28 k_{B} T$ (for $\Omega_{\mathrm{W}}=1$ ), the water molecule $\mathrm{W} 1$ prefers to maintain its bound state with $\mathrm{K} 1$ and follows the preceding ion K1 towards the site $S_{1}$. This type of movement was also observed during our MD simulations (Fig. 4). The translocations of K1 and W1 create a vacancy at the binding site $S_{2}$ which is used in the next hopping event by the ion $\mathrm{K} 2$ from $\mathrm{S}_{3}$ to $\mathrm{S}_{2}$. A backward motion of W1 to $S_{2}$ is almost prohibited by the high energy barrier imposed by the pore potential on W1. Indeed, the 
present MD simulations (and unpublished data) did not provide any significant evidence for a retrograde translocation of W1. Similar observations have been reported recently from studies of long artificial nanochannels which are occupied by an alternating sequence of ions and water. ${ }^{24}$

\section{Is there a ratchet effect by permions?}

In the following paragraph, we want to address two specific aspects of our MD simulations and the lattice model.

First, it is of interest to note that our observations of coupled ion-water movements could eventually be described by the recently proposed concept of permions, ${ }^{21}$ where the latter is a quasiparticle which should describe the collective cooperative movements of the constituents of the pore, i.e., the backbone atoms, ions, and water molecules. Similar as in solid state theory, the permion should take into account the many body character of the transport process. It would be a challenging task to develop a mathematical theory of permions.

Second, if the observed absence of retrograde movements of ions and water molecules in the pore could be attributed to a significant difference in the energy barriers of the pore potential with respect to ions and water molecules, then a hypothetical permion, where one ion and one water molecule are in a bound state, would be an asymmetric quasiparticle. In this case the water molecule would effectively act as a rectifier. It is known that a single particle confined in an asymmetric potential exhibits an anticipated ratchet effect by drifting along the "easy" ratchet direction when subjected to nonequilibrium fluctuations. ${ }^{42-45}$ In the present case, however, this concept has to be modified since the asymmetry is not due to the potential, but due to the asymmetry of the quasiparticle. The concept of localized ratchet has been employed in a recent theory by Lee and Sung ${ }^{19}$ in order to take into account effects of nonequilibrium fluctuations on ionic transport through ion channels. Whether this theoretical ansatz could be used to explain the transport specifically in potassium ion channels is still to be demonstrated.

In this context it may be of interest to point out the important role of the water molecules intercalated between the ions inside the pore. Without water, the ion transport would correspond approximately to the case of superionic conductors, which is described by the Frenkel-Kontorova model. ${ }^{46-49}$

Therefore, the ion conduction in potassium ion channels seems to be indeed a water-mediated transport process.

\section{CONCLUSIONS}

The present study reports on an attempt to provide a microscopic concept of transport in potassium ion channels. Therefore, we have performed molecular dynamics simulations of the KcsA potassium channel. Employing a simulation technique similar to atomic force microscopy, we have observed collective and cooperative movements of ions, water molecules, and the backbone atoms of the pore. A detailed analysis of these movements leads to the development of a simple theoretical model of ion-water transport. The transport can be described by pairwise hopping of ion-water pairs mediated by the potential of the backbone atoms of the pore. It is suggested that the function of the water molecules is probably to serve as rectifiers during the ion-water movements.

In order to corroborate our results and interpretations, it would be useful to investigate other types of potassium channels as well. Our results are based on a single pulling velocity and on three MD trajectories only, which cannot be considered as a statistical ensemble. However, we assume that more simulations would increase the accuracy of our results, but would not change essentially our conclusions.

\section{ACKNOWLEDGMENTS}

We gratefully thank Dr. Jung-Hsing Lin for the help on the model construction and for many useful suggestions. The computer simulations were done on the supercomputers of Zentralinstitut für Angewandte Mathematik (ZAM) of Forschungszentrum Jülich.

${ }^{1}$ B. Hille, Ion Channels of Excitable Membranes, 3rd ed. (Sinauer Associates Inc., Sunderland, Massachusetts, 2001).

${ }^{2}$ J. H. Morais-Cabral, Y. F. Zhou, and R. MacKinnon, Nature (London) 414, 37 (2001).

${ }^{3}$ Y. Zhou and R. MacKinnon, J. Mol. Biol. 333, 965 (2003).

${ }^{4}$ Y. F. Zhou, J. H. Morais-Cabral, A. Kaufman, and R. MacKinnon, Nature (London) 414, 43 (2001).

${ }^{5}$ B. Hille and W. Schwarz, J. Gen. Physiol. 72, 409 (1978).

${ }^{6}$ A. L. Hodgkin and R. D. Keynes, J. Physiol. (London) 128, 61 (1955).

${ }^{7}$ S. Kuyucak, S. Andersen, and S.-H. Chung, Rep. Prog. Phys. 64, 1427 (2001).

${ }^{8}$ S.-H. Chung, T. W. Allen, and S. Kuyucak, Biophys. J. 82, 628 (2002).

${ }^{9}$ R. Marshl, Y. Tang, J. Schnitzer, and E. Jacobsson, Biophys. J. 81, 2473 (2001).

${ }^{10}$ A. Burykin, C. N. Schutz, J. Villa, and A. Warshel, Proteins: Struct., Funct., Genet. 47, 265 (2002).

${ }_{11}^{11}$ J. Åqvist and V. Luzhkov, Nature (London) 404, 881 (2000).

${ }^{12}$ S. Bernéche and B. Roux, Nature (London) 414, 73 (2001).

${ }^{13}$ S. Berneche and B. Roux, Proc. Natl. Acad. Sci. U.S.A. 100, 8644 (2003).

${ }^{14}$ C. Domene and M. S. P. Sansom, Biophys. J. 85, 2787 (2003).

${ }^{15}$ S. Garofoli and P. C. Jordan, Biophys. J. 84, 2814 (2003).

${ }^{16}$ P. Läuger, Biochim. Biophys. Acta 311, 423 (1973).

${ }^{17}$ K. Cooper, P. Gates, and R. Eisenberg, J. Membr. Biol. 106, 95 (1988).

${ }^{18}$ K. Cooper, P. Gates, and R. Eisenberg, Q. Rev. Biophys. 21, 331 (1988).

${ }^{19}$ K. Lee and W. Sung, Phys. Rev. E 60, 4681 (1999).

${ }^{20}$ K. Lee and W. Sung, Physica A 315, 79 (2002).

${ }^{21}$ R. Elber, D. P. Chen, D. Rojewska, and R. Eisenberg, Biophys. J. 68, 906 (1995).

${ }^{22}$ H. Grubmüller, B. Heymann, and P. Tavan, Science 271, 997 (2001).

${ }^{23}$ J. R. Gullingsrud, R. Braun, and K. Schulten, J. Comput. Phys. 151, 190 (1999).

${ }^{24}$ J. F. Gwan and A. Baumgaertner, J. Comput. Theor. Nanosci. 4, 50 (2007).

${ }^{25}$ D. A. Doyle, J. Morais-Cabral, R. A. Pfuetzner, A. Kuo, J. M. Gulbis, S. L. Cohen, B. T. Chait, and R. MacKinnon, Science 280, 69 (1998).

${ }^{26}$ V. Luzhkov and J. Åqvist, Biochim. Biophys. Acta 1481, 360 (2000).

${ }^{27}$ S. Berneche and B. Roux, Biophys. J. 82, 772 (2002)

${ }^{28}$ K. Ranatunga, I. H. Shrivastava, G. R. Smith, and M. S. P. Sansom, Biophys. J. 80, 1210 (2001)

${ }^{29}$ M. Compoint, C. Ramseyer, and P. Huetz, Chem. Phys. Lett. 397, 510 (2004).

${ }^{30}$ H. Heller, M. Schaefer, and K. Schulten, J. Phys. Chem. 97, 8343 (1993).

${ }^{31}$ J.-H. Lin and A. Baumgaertner, Biophys. J. 78, 1714 (2000).

${ }^{32}$ B. Isralewitz, M. Gao, and K. Schulten, Curr. Opin. Struct. Biol. 11, 224 (2001).

${ }^{33}$ D. A. Case, D. A. Pearlman, J. W. Caldwell et al., AMBER 5.0, University of California, San Francisco, 1997.

${ }^{34}$ H. J. C. Berendsen, J. P. M. Postma, W. F. van Gunsteren, A. DiNola, and 
J. R. Haak, J. Chem. Phys. 81, 3684 (1984).

${ }^{35}$ S. Harvey, R.-Z. Tan, and T. III, J. Comput. Chem. 19, 726 (1998).

${ }^{36}$ U. Essmann, L. Perera, M. L. Berkowitz, T. Darden, H. Lee, and L. G. Pedersen, J. Chem. Phys. 103, 8577 (1995).

${ }^{37}$ S. J. Weiner, P. A. Kollman, D. T. Nguyen, and D. A. Case, J. Comput. Chem. 7, 230 (1986).

${ }^{38}$ K. Kearsley, Acta Crystallogr., Sect. A: Found. Crystallogr. 45, 208 (1989).

${ }^{39}$ S. Bernéche and B. Roux, Biophys. J. 78, 2900 (2000).

${ }^{40}$ I. H. Shrivastava and M. S. P. Sansom, Biophys. J. 78, 557 (2000).

${ }^{41}$ R. Gabler, Electrical Interactions in Molecular Biophysics, 1st ed. (Aca- demic, New York, 1978).

${ }^{42}$ M. von Smoluchowski, Phys. Z. 13, 1069 (1912).

${ }^{43}$ R. Feynman, R. Leighton, and M. Sands, The Feynman Lectures on Physics (Addison Wesley, Reading, MA, 1963), Vol. 1, Chap. 46.

${ }^{44}$ M. O. Magnasco, Phys. Rev. Lett. 71, 1477 (1993).

${ }^{45}$ A. Ajdari and J. Prost, C.R. Acad. Sci., Ser. IIc: Chim 315, 1635 (1992).

${ }^{46}$ J. C. Wang and D. F. Pickett, J. Chem. Phys. 65, 5378 (1976).

${ }^{47}$ J. B. Sokoloff and A. Widom, Phys. Rev. B 18, 2824 (1978).

${ }^{48}$ T. Geisel, Phys. Rev. B 20, 4294 (1979).

${ }^{49}$ H. U. Beyeler, L. Pietronero, and S. Strässler, Phys. Rev. B 22, 2988 (1980). 